\title{
Caffeine-induced food-avoidance behavior is mediated by neuroendocrine signals in Caenorhabditis elegans
}

\author{
Hyemin Min ${ }^{\#}$, Esther Youn ${ }^{\#}$, Ichiro Kawasaki \& Yhong-Hee Shim ${ }^{*}$ \\ Department of Bioscience and Biotechnology, Konkuk University, Seoul 05029, Korea
}

\begin{abstract}
High-dose caffeine uptake is a developmental stressor and causes food-avoidance behavior (aversion phenotype) in $C$. elegans, but its mode of action is largely unknown. In this study, we investigated the molecular basis of the caffeineinduced aversion behavior in C. elegans. We found that aversion phenotype induced by $30 \mathrm{mM}$ caffeine was mediated by JNK/MAPK pathway, serotonergic and dopaminergic neuroendocrine signals. In this process, the dopaminergic signaling appears to be the major pathway because the reduced aversion behavior in cat-2 mutants and mutants of JNK/MAPK pathway genes was significantly recovered by pretreatment with dopamine. RNAi depletion of $h s p-16.2$, a cytosolic chaperone, and cyp-35A family reduced the aversion phenotype, which was further reduced in cat-2 mutants, suggesting that dopaminergic signal is indeed dominantly required for the caffeine-induced food aversion. Our findings suggest that aversion behavior is a defense mechanism for worms to survive under the high-dose caffeine conditions. [BMB Reports 2017; 50(1): 31-36]
\end{abstract}

\section{INTRODUCTION}

Caffeine is one of the most widely used drugs in the world as a part of consumed food. Caffeine uptake induces both beneficial and adverse effects on a body in a dosage- and developmental stage-dependent manner $(1,2)$. A moderate dose of caffeine uptake has a neuroprotective power and is also beneficial to physical performance in human as previously described (3). However, overconsumption of caffeine can be harmful to human health. Although the effects of caffeine can be lethal, the mode of its action in the body is still

*Corresponding author. Tel: +82-2-450-4059; Fax: +82-2-4559956; E-mail: yshim@konkuk.ac.kr

${ }^{\#}$ These authors contributed equally to this work.

https://doi.org/10.5483/BMBRep.2017.50.1.126

Received 26 July 2016, Revised 17 August 2016, Accepted 29 September 2016

Keywords: Caenorhabditis elegans, Caffeine, Food-avoidance behavior, JNK/MAPK pathway, Neuroendocrine signals unclear.

In our previous study, we investigated effects of caffeine on Caenorhabditis elegans. C. elegans is an excellent model organism to analyze the effects of bioactive molecules on animal behaviors. We found that an overdose $(30 \mathrm{mM})$ of caffeine treatment inhibited the early larval development and induced a stress response by up-regulating heat shock proteins, suggesting that high-dose caffeine is a developmental stressor (4). Interestingly, we also found that the food-aversion phenotype (food-avoidance behavior) was induced by the caffeine treatment, which was abolished by RNAi depletion of $h s p-16.2$ (4). HSP-16.2 is a small heat shock inducible cytosolic chaperone protein in C. elegans (5). The above finding suggests that the aversion phenotype is a defensive behavior for animal survival in response to an overdose of caffeine.

In this study, we further investigated whether the caffeineinduced aversion behavior is mediated by a neuronal or non-neuronal tissue response. Here we report that the food aversion phenotype observed after treatment with $30 \mathrm{mM}$ of caffeine is mediated by neuroendocrine signals, in which dopamine serves as a dominant signaling and serotonin serves as a supplementary signaling. Further we show that these neuroendocrine signals are associated with cellular stress responses mediated by jnk-1, hsp-16.2, cyp-35A2, and cyp-35A4.

\section{RESULTS}

Caffeine induced food-avoidance behavior (aversion phenotype) in a dosage and developmental stage-dependent manner

We found that the level of food avoidance (\% aversion) was caffeine-dose dependent. Among them, $30 \mathrm{mM}$ caffeine treatment at the L1 larval stage caused $18.0 \pm 4.5 \%$ of food aversion (Fig. 1A). However, caffeine treatment at the later larval stages, L2 and L3 showed more reduced levels of aversion than L1, and caffeine treatment at the L4 larval stage did not cause significant food aversion phenotype (Fig. 1A). In our previous study, we reported that high-dose caffeine induced developmental growth retardation when treated at early larval stages (2). As in caffeine-induced growth retardation, we observed that early-stage larvae were more sensitive to caffeine for aversion behavior than later-stage larvae (Fig. 1A). We also reported that cyp-35A gene family, 


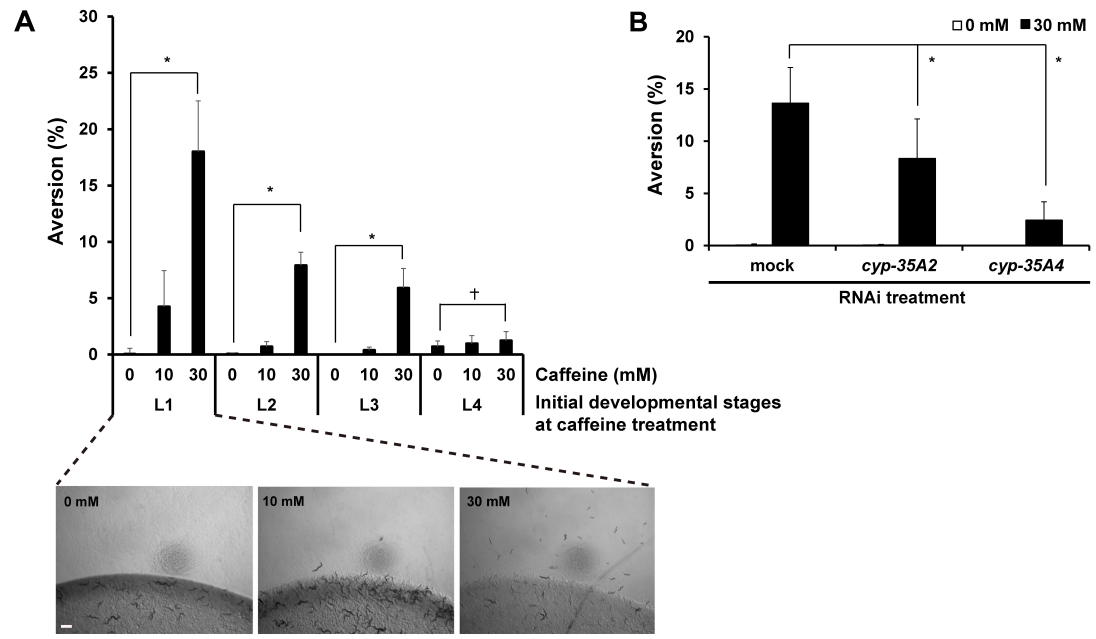

Fig. 1. Caffeine induced aversion phenotype in C. elegans. (A) Wild-type worms synchronized at the $L 1$ larval stage $(0 \mathrm{mM}$, $\mathrm{n}=673 ; 10$ $\mathrm{mM}, \mathrm{n}=535 ; 30 \mathrm{mM}, \mathrm{n}=565)$, L2 larval stage $(0 \mathrm{mM}, \mathrm{n}=649 ; 10 \mathrm{mM}, \mathrm{n}=531 ; 30 \mathrm{mM}, \mathrm{n}=536)$, L3 larval stage $(0 \mathrm{mM}, \mathrm{n}=847 ;$ $10 \mathrm{mM}, \mathrm{n}=348 ; 30 \mathrm{mM}, \mathrm{n}=487)$, and L4 larval stage $(0 \mathrm{mM}, \mathrm{n}=249 ; 10 \mathrm{mM}, \mathrm{n}=322 ; 30 \mathrm{mM}, \mathrm{n}=306)$ were cultured on NGM agar plates containing 0,10 , or $30 \mathrm{mM}$ caffeine, and their aversion phenotype was observed $24 \mathrm{~h}$ after caffeine treatment at $20^{\circ} \mathrm{C}$. Representative images of worms treated with 0,10 or $30 \mathrm{mM}$ caffeine when they were at the L1 larval stage are shown under the graph (B) The caffeine-induced aversion phenotype after RNAi depletion of cyp-35A2 and cyp-35A4 in wild type. After RNAi depletion, the synchronized L1 larvae were cultured on NGM agar plates containing 0 or $30 \mathrm{mM}$ caffeine, and the aversion phenotype was observed at $24 \mathrm{~h}$ after the caffeine treatment (mock RNAi, $\mathrm{n}=763 ;$ cyp-35A2 RNAi, $\mathrm{n}=823 ;$ cyp-35A4 RNAi, $\mathrm{n}=654$ ). In (A, B), values are shown as average $\%$ food aversion from three independent experiments. Bar, $200 \mu \mathrm{m}$. Error bars represent s.d. ${ }^{*} \mathrm{P}<0.05$. ${ }^{+} \mathrm{P}>0.05$.

the detoxification pathway genes were highly induced after 30 $\mathrm{mM}$ caffeine treatment, and that RNAi depletion of these genes showed partial recovery from growth retardation caused by caffeine treatment (2). Therefore, we examined whether RNAi depletion of cyp-35 genes also affects the aversion phenotype. In fact, we found that RNAi of cyp-35A2 and cyp-35A4 caused lower levels of aversion than mock RNAi control (Fig. 1B). This result suggests that growth retardation induced by $30 \mathrm{mM}$ caffeine was affected by food aversion.

\section{Caffeine-induced food-avoidance behavior is mediated by JNK/MAPK pathway}

To further investigate the correlation between food avoidance and growth retardation, we next examined MAPK signaling pathway, which is required for microbial aversion (6), and RNAi depletion of MAPK pathway genes was previously reported to cause growth retardation (wormbase.org). We examined food aversion behavior under 0 or $30 \mathrm{mM}$ caffeine condition, after RNAi depletion of genes involved in the MAPK pathway. We found that the aversion phenotype was suppressed when tir-1, sek-1, pmk-1, mlk-1, mek-1, vhp-1, $k g b-1, j k k-1$ and $j n k-1$ genes were depleted under $30 \mathrm{mM}$ caffeine conditions compared to mock RNAi control (Fig. 2A: mock RNAi, $11.0 \pm 0.4 \%$; tir-1 RNAi, $4.4 \pm 0.4 \%$; sek-1 RNAi, $6.3 \pm 1.1 \%$; pmk-1 RNAi, $5.6 \pm 1.4 \%$; mlk-1 RNAi, $7.5 \pm 1.2 \%$; mek-1 RNAi, $3.5 \pm 1.6 \%$; vhp-1 RNAi, $5.1 \pm$ $0.6 \%$; kbg-1 RNAi, $3.9 \pm 0.3 \%$; jkk-1 RNAi, $2.9 \pm 1.0 \%$; jnk-1 RNAi, $3.8 \pm 0.4 \%$ ). Among them, RNAi depletion of tir-1, mek-1, kgb-1, jkk-1, and jnk-1 reduced the aversion phenotype more effectively than the other genes (Fig. 2A). We also tested caffeine-induced aversion phenotype using genetic loss-of-function mutants of $m / k-1$, mek-1, kgb-1, and jnk-1 genes. The results obtained using the loss-of-function mutants were similar to those obtained by RNAi depletion (Fig. 3A). We judge that efficiency of RNAi depletion in this study was high enough to evaluate deficiency of tested gene activities. These results suggest that activation of MAPK pathway genes is collectively required for caffeine-induced food aversion. Among the MAPK pathway genes, JNK kinase pathway genes appear to have a major role for the control of caffeine-induced food aversion behavior. Furthermore, the reduced level of food avoidance we observed in loss-of-function mutants of MAPK pathway genes was unlikely related to growth retardation.

Caffeine-induced food-avoidance behavior is mediated by both serotonin and dopamine signals, in which dopamine has a major role

To investigate a possible correlation between caffeine-induced food-avoidance behavior and a neuronal signaling, we observed the aversion phenotype after caffeine treatment in dopamine-synthesis defective cat-2, serotonin-synthesis defective tph-1, and both serotonin- and dopamine-synthesis defective bas- 1 mutants. We found that the aversion phenotype was reduced significantly in cat-2, tph-1, and more significantly in 

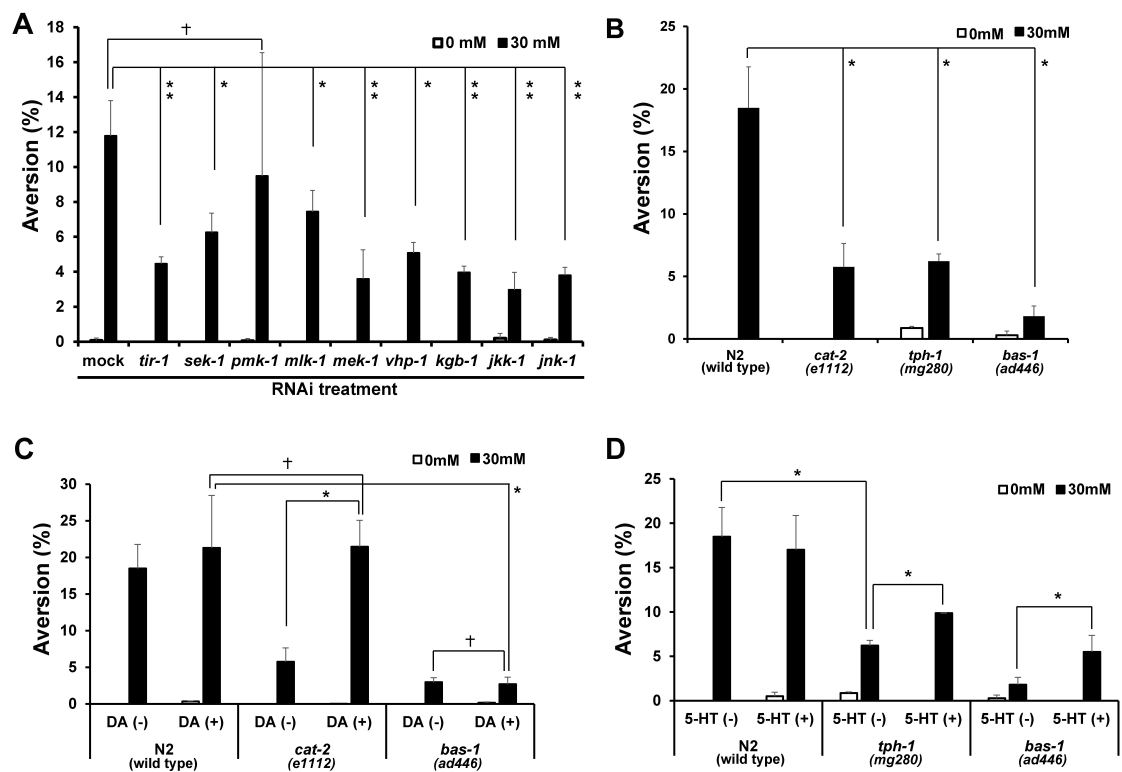

Fig. 2. The JNK/MAPK pathway and neuronal signaling were required for caffeine-induced aversion phenotype. (A) The JNK/MAPK pathway mediates caffeine-induced aversion phenotype demonstrated by RNAi depletion of tir-1, sek-1, pmk-1, mlk-1, mek-1, vhp-1, kgb-1, jkk-1 and jnk-1 in N2 worms. After RNAi depletion, synchronized L1 larvae were cultured on NGM agar plates containing 0 or $30 \mathrm{mM}$ caffeine, and the aversion phenotype was observed at $24 \mathrm{~h}$ after caffeine treatment (mock RNAi, $\mathrm{n}=894$; tir-1 RNAi, $\mathrm{n}=821$; sek-1 $\mathrm{RNAi}_{1} \mathrm{n}=797 ;$ pmk-1 RNAi, $\mathrm{n}=877 ; m / k-1 \mathrm{RNAi}, \mathrm{n}=676 ;$ mek-1 RNAi, $\mathrm{n}=874 ;$ vhp-1 RNAi, $\mathrm{n}=809 ; k g b-1$ RNAi, $\mathrm{n}=781$; $j k k-1$ RNAi, $\mathrm{n}=1020$; jnk-1 RNAi, $\mathrm{n}=898$ ). (B) Dopamine-deficient cat-2, serotonin-deficient tph-1, serotonin and dopamine-deficient bas-1 mutants showed defects in food aversion at $30 \mathrm{mM}$ caffeine. cat-2, tph-1 and bas-1 mutant worms synchronized at the L1 larval stage were cultured on NGM agar plates containing 0 or $30 \mathrm{mM}$ caffeine, and the aversion phenotype was observed at $24 \mathrm{~h}$ after the caffeine treatment $(\mathrm{N} 2, \mathrm{n}=589 ;$ cat-2, $\mathrm{n}=380 ;$ tph-1, $\mathrm{n}=966 ;$ bas-1, $\mathrm{n}=623)$. (C, D) $\mathrm{N} 2$, cat-2, tph-1 and bas-1 worms synchronized at the L1 larval stage were pre-treated for $10 \mathrm{~min}$ with dopamine (DA) or serotonin (5-HT), cultured on NGM agar plates containing 0 or $30 \mathrm{mM}$ caffeine, and the aversion phenotype was monitored at $24 \mathrm{~h}$ after caffeine treatment $(\mathrm{N} 2, \mathrm{n}=793 ;$ cat-2, $\mathrm{n}=$ 511; tph-1, $\mathrm{n}=763$; bas-1, $\mathrm{n}=524)$. Values are shown as average $\%$ food aversion. Error bars represent s.d. ${ }^{*} \mathrm{P}<0.05$. ${ }^{* * P}<$ $0.005 .{ }^{\dagger} \mathrm{P}>0.05$.

bas-1 mutants when worms were exposed to $30 \mathrm{mM}$ caffeine (Fig. 2B: N2, $18.4 \pm 3.2 \%$; cat-2, $5.8 \pm 1.8 \%$; tph-1, $6.2 \pm$ $0.6 \%$; bas- $1,1.8 \pm 0.8 \%$ ). These results suggest that both dopamine and serotonergic signaling pathways are required for the food avoidance after caffeine treatment. The defect of food-avoidance behavior in cat-2 mutants, but not bas- 1 mutants, was sufficiently rescued by acute-dopamine treatment (Fig. 2C). However, the caffeine-induced aversion phenotype was not sufficiently rescued by acute-serotonin treatment in neither tph-1 nor bas-1 mutants (Fig. 2D). These results suggest that although both dopaminergic and serotonergic signals are required for the aversion phenotype, dopaminergic signaling is the major pathway for controlling the aversion phenotype in the presence of high-dose caffeine. To confirm that these signaling pathways are active at the L1 larval stage, we monitored expression of dat-1::GFP dopaminergic transgene and tph-1::GFP serotonergic transgene in the L1 stage transgenic worms. We indeed confirmed that both dat-1::GFP and tph-1::GFP were highly expressed at the L1 larval stage (Fig. S1).

\section{JNK kinase signaling possibly functions upstream of dopaminergic neuronal signaling to control caffeine-induced food-avoidance behavior}

To test whether the reduced caffeine-induced aversion phenotype in mutants of JNK/MAPK pathway genes are restored by acute-dopamine and serotonin treatment, we measured the caffeine-induced aversion phenotype in wild-type and mutants of JNK/MAPK pathway genes with or without DA or 5-HT treatment. We hypothesized that if JNK/MAPK signaling pathway is acting upstream of dopaminergic or serotonergic signaling the reduced aversion phenotype in mutants of JNK/MAPK signaling pathway genes is rescued by either dopamine or serotonin treatment. We found that aversion phenotype was rescued by neither DA nor 5-HT treatment in kgb-1 mutant and mek-1 mutants while the aversion phenotype was partially rescued by DA, but not noticeably by 5- $\mathrm{HT}$, in $m / k-1$ and jnk-1 mutants (Fig 3A: mlk-1: control, 3.5 $\pm 0.3 \%$, with DA, $5.5 \pm 0.8 \%$; jnk-1: control, $0 \%$, with DA, $6.8 \pm 1.1 \%)$. Remarkably, the caffeine-induced aversion phenotype that was almost completely suppressed in jnk-1 

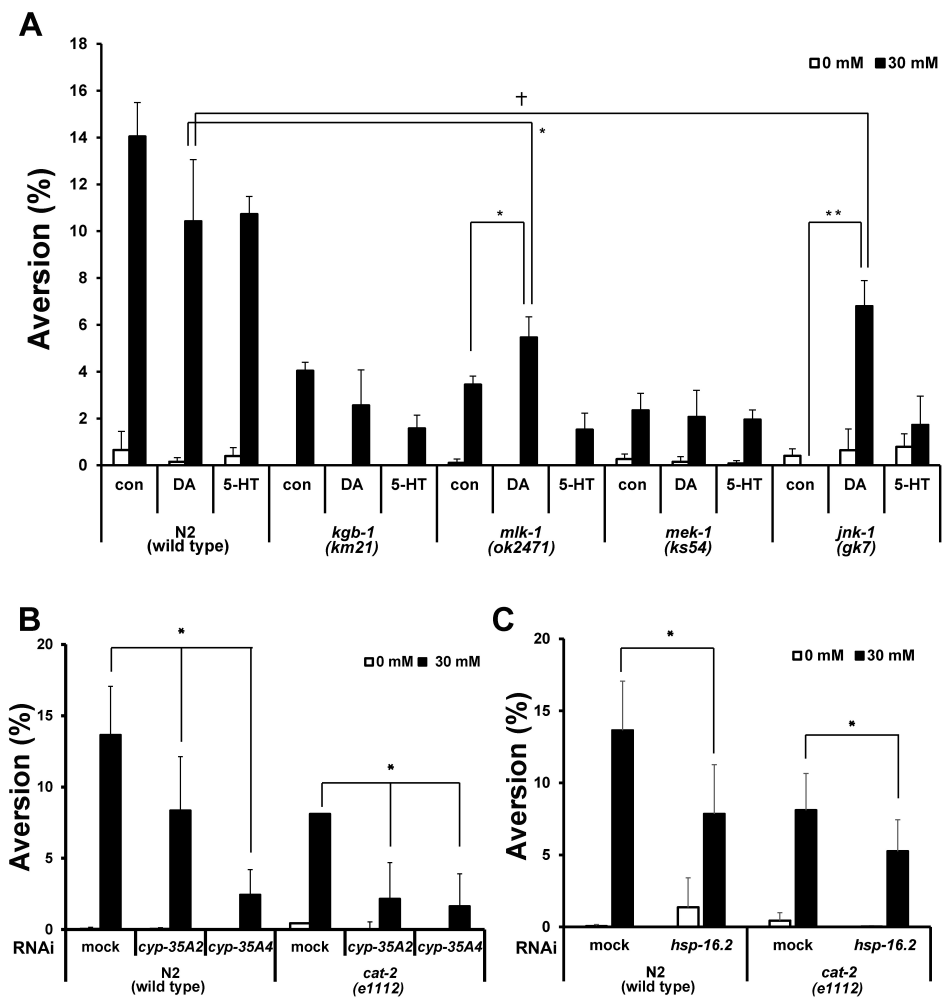

Fig. 3. Caffeine-induced food-avoidance behavior was mediated by JNK/MAPK and neuroendocrine signaling. (A) Aversion phenotype in JNK/MAPK mutants (kgb-1, mlk-1, mek-1, jnk-1) after $0 \mathrm{mM}$ or $30 \mathrm{mM}$ caffeine treatment with or without acute-dopamine or serotonin treatment. Worms synchronized at the L1 larval stage were cultured on NGM agar plates containing 0 or $30 \mathrm{mM}$ caffeine, and the aversion phenotype was observed at $24 \mathrm{~h}$ after the caffeine treatment $(\mathrm{N} 2, \mathrm{n}=4099 ; k g b-1, \mathrm{n}=1559 ; m / k-1, \mathrm{n}=1638 ; m e k-1, \mathrm{n}=$ 1704; jnk-1, $\mathrm{n}=929$ ). Values are shown as average \% food aversion \pm s.d. (B) Aversion phenotype after RNAi depletion of cyp-35A2, cyp-35A4 in N2 and cat-2 mutants. After the RNAi depletion, the synchronized L1 larvae were cultured on NGM agar plates containing 0 or $30 \mathrm{mM}$ caffeine, and the aversion phenotype was observed at $24 \mathrm{~h}$ after caffeine treatment (individual RNAi, $\mathrm{n}>654$ ). (C) Aversion phenotype after RNAi depletion of $h s p-16.2$ in N2 and cat-2 mutants. After the RNAi depletion, the synchronized L1 larvae were cultured on NGM agar plates containing 0 or $30 \mathrm{mM}$ caffeine, and the aversion phenotype was observed at $24 \mathrm{~h}$ after caffeine treatment (individual RNAi, $\mathrm{n}>723$ ). Values are shown as average $\%$ food aversion. Error bars represent $\mathrm{s.d}$. ${ }^{*} \mathrm{P}<0.05$. ${ }^{\dagger} \mathrm{P}>0.05$.

mutants was significantly recovered by dopamine, but not by serotonin, treatment (Fig. 3A). These results indicate that JNK pathway has a critical role for controlling caffeine-induced aversion phenotype, and that this pathway is possibly acting upstream of dopaminergic signaling to control caffeineinduced food-avoidance behavior.

We previously reported that expression of cyp-35A family genes and $h s p-16.2$, a cytosolic chaperone, was induced by caffeine treatment and that RNAi depletion of hsp-16.2 reduced the caffeine-induced aversion phenotype (4). In this study, we also showed that expression of cyp-35::GFP transgene was induced by caffeine treatment in the L1 larval stage of transgenic worms (Fig. S2). In addition, we showed that RNAi depletion of cyp-35A2 and cyp-35A4 also reduced the aversion phenotype (Fig. 1B). These findings suggest that high expression of cyp-35A family and $h s p-16.2$ genes induces the aversion phenotype in response to a toxic environment induced by high-dose caffeine. We further examined whether the reduced level of food aversion after RNAi depletion of сур-35A2, сyp-35A4, or hsp-16.2 is further reduced in cat-2 mutants. We found that the reduction of food aversion behavior after RNAi depletion of cyp-35A2, cyp-35A4, and hsp-16.2 was more substantial in $30 \mathrm{mM}$-caffeine treated cat-2 mutants than in $30 \mathrm{mM}$-caffeine treated wild-type $\mathrm{N} 2$ animals (Fig. 3B, 3C). These findings suggest two possibilities: the caffeine-induced aversion phenotype which is enhanced by expression of cyp-35A2, cyp-35A4, and hsp-16.2 genes, requires dopaminergic signal pathway; or the stress-responding cyp-35A2, сур-35A4, and hsp-16.2 genes and the dopaminergic signal pathway are acting in parallel, so that when both pathways were depleted, the defect in food aversion was observed additively. 


\section{DISCUSSION}

A food-avoidance behavior is considered as one of defense mechanisms from harsh environments. Our previous study revealed that high-dose caffeine treatment induces HSP-16.2, a small heat shock inducible cytosolic chaperone protein and RNAi depletion of $h s p-16.2$ abolished the food-aversion behavior (4). This result suggests that HSP-16.2 is required for the food-avoidance behavior and that this behavior is a program for survival. HSP-16.2 was expressed under stressful conditions, and the expression of HSP-16.2 was associated with nuclear translocation of DAF-16, which is a transcriptional activator of HSP-16.2 (7). This finding suggests that survival pathway via HSP-16.2 and the aversion behavior is possibly coupled with life-span control of worms. In this study we also revealed that RNAi of cyp-35A2 (cytochrome P450) and cyp-35A4, which are highly induced by caffeine treatment (2), reduced the food-avoidance behavior, suggesting that a detoxification pathway mediated by cyp-35A2 and cyp-35A4 is also required for inducing the food-avoidance behavior after caffeine treatment.

Based on these findings, we further investigated underlying molecular mechanism that controls the caffeine-induced food-avoidance behavior. We hypothesized that high-dose caffeine uptake triggers changes in normal cellular processes and stimulates the food-avoidance behavior. It was previously reported that a disruption of cellular activities induced a food-avoidance behavior which was controlled by serotonergic and JNK/MAPK kinase signaling pathways (6). Similarly, our results revealed that JNK/MAPK pathway, and serotonergic and dopaminergic neuroendocrine signals are required for food-avoidance behavior induced by caffeine treatment, in which dopaminergic signaling seems to be the major pathway to respond to caffeine treatment. Lack of neuroendocrine signals abolished or significantly suppressed the food-avoidance behavior, which was substantially recovered by providing the signals exogenously. These results indicate that production of the neuroendocrine signals is essential for the food-avoidance behavior induced by caffeine treatment. In addition, it was reported that eating behaviors involved dopaminergic neural circuitry which affected the food- and drug-related cues in the brain $(8,9)$. This idea is supported by the findings that well conserved dopamine biosynthesis and transport pathways are required to maintain homeostasis in humans, Drosophila and C. elegans (10). Recently, it was reported that caffeine suppresses nighttime sleep through dopamine signaling in Drosophila (11). Nevertheless, thus far, transcription factors, which are involved in dopamine signaling after caffeine treatment, remain to be determined. Taken together, high-dose caffeine treatment to $C$. elegans has adverse effects on the development, and induces a food-avoidance behavior for the survival. For this response the JNK/MAPK pathway is required. In addition, the neuroendocrine signals, serotonin and dopamine are also required. Caffeine has various beneficial effects on the body when appropriate doses are administered. However, overdose of caffeine is a stressor to the body and induces a survival pathway, which results in changes of various cellular activities. Especially, caffeine consumption at early developmental stages can be lethal through inducing growth retardation and severe food avoidance.

\section{MATERIALS AND METHODS}

\section{C. elegans strains and maintenance}

All strains were maintained at either $15^{\circ} \mathrm{C}$ or $20^{\circ} \mathrm{C}$ on Nematode Growth Medium (NGM) agar plates seeded with Escherichia coli OP50 as previously described (12). The following strains were used: CB1112: cat-2(e1112) II, MT7988: bas-1(ad446) III, CY573: vbls5[cyp-35B1p::GFP + gсy-7p::GFP]?, FK171: mek-1(ks54) X, KU21: kgb-1(km21) IV, MT15434: tph-1(mg280) II, RB1908: mlk-1(ok2471) V, VC8: jnk-1(gk7) IV, BZ555: eg/s1 [dat-1p::GFP]?, GR1366: mgls42 [tph-1::GFP + rol-6(su1006)]?. N2 was used as a wild type. For caffeine treatment, the appropriate amount of caffeine (Sigma, St. Louis, MO, USA) was included in the NGM agar plates before autoclaving to achieve final concentration of $30 \mathrm{mM}$.

\section{RNA interference (RNAi) analysis}

RNAi analysis was performed using the "RNAi-by-soaking" method as described previously (13). Wild-type worms were synchronized at the L1 larval stage in absence of food, and soaked for $24 \mathrm{~h}$ in each of the double-strand RNA solutions transcribed in vitro from respective cDNA templates, which were PCR-amplified from corresponding L4440 feeding RNAi clones using T7 primer, 5'-GTAATACGACTCACTATAGGGC3', and L4440 T7 primer, 5'-ATTAATACGACTCACTATAG GGA-3'. Worms were then transferred to either NGM plates containing caffeine or control NGM plates at $20^{\circ} \mathrm{C}$. Aversion phenotype was observed at $24 \mathrm{~h}$ after the transfer of the worms. Efficiency of RNAi was examined by final phenotype of individual test genes.

\section{Food-avoidance behavior (aversion phenotype) assay}

To check for food avoidance after caffeine treatment, synchronized L1 worms were transferred to E. coli OP50-seeded NGM plates with or without $30 \mathrm{mM}$ caffeine. Food avoidance of worms was calculated by dividing the number of worms that stayed outside of food area with the total number of worms on the plate as described previously (6). Each RNAi or drug treatment was conducted in triplicate to evaluate statistical significance of the data, unless otherwise indicated.

\section{Acute dopamine and serotonin treatment}

Dopamine (Sigma) and serotonin (Sigma) solutions were prepared in M9 buffer (3.0 g KH $\mathrm{KO}_{4}, 6.0 \mathrm{~g} \mathrm{Na} \mathrm{HPO}_{4}, 0.5 \mathrm{~g}$ $\mathrm{NaCl}, 1.0 \mathrm{~g} \mathrm{NH}_{4} \mathrm{Cl}$ in $1 \mathrm{~L} \mathrm{H}_{2} \mathrm{O}$ ). Synchronized $\mathrm{L} 1$ worms were treated with dopamine $(3 \mathrm{mg} / \mathrm{ml}, 15 \mathrm{mM})$ or serotonin $(3$ 
$\mathrm{mg} / \mathrm{ml}, 7.4 \mathrm{mM}$ ) for $10 \mathrm{~min}$, based on previous reports (14, 15). The optimal conditions for acute dopamine and serotonin treatments were determined by finding the concentration for the most effective rescue from the food aversion defect (Fig. S3). After the drug treatments, worms were transferred to either caffeine-containing or control NGM plates at $20^{\circ} \mathrm{C}$. Control worms were treated with M9 buffer.

\section{Statistical analysis}

All experiments were repeated more than three times for statistical evaluation of data. $P$ values were calculated by Student's t-test. We considered $\mathrm{P}<0.05$ as being significant.

\section{ACKNOWLEDGEMENTS}

Nematode strains were provided by the Caenorhabditis Genetics Center, which is funded by NIH Office of Research Infrastructure Programs (P40 OD010440). This study was supported by fund from Konkuk University in 2013 provided to Y.-H. Shim.

\section{CONFLICTS OF INTEREST}

The authors have no conflicting financial interests.

\section{REFERENCES}

1. Sutphin GL, Bishop E, Yanos ME, Moller RM and Kaeberlein M (2012) Caffeine extends life span, improves healthspan, and delays age-associated pathology in Caenorhabditis elegans. Longev Healthspan 1, 1-9

2. Min H, Kawasaki I, Gong J and Shim YH (2015) Caffeine induces high expression of cyp-35A family genes and inhibits the early larval development in Caenorhabditis elegans. Mol Cells 38, 236-242

3. Cabrera C, Artacho R and Giménez R (2006) Beneficial effects of green tea-a review. J Am Coll Nutr 25, 79-99

4. Al-Amin M, Kawasaki I, Gong J and Shim YH (2016) Caffeine induces the stress response and up-regulates heat shock proteins in Caenorhabditis elegans. Mol Cells 39, 164-168

5. Fonte V, Kipp DR, Yerg J et al (2008) Suppression of in vivo beta-amyloid peptide toxicity by overexpression of the HSP-16.2 small chaperon protein. J Biol Chem 283, 784-791

6. Melo JA and Ruvkun G (2012) Inactivation of conserved C. elegans genes engages pathogen- and xenobioticassociated defenses. Cell 149, 452-466

7. Hartwig K, Heidler T, Moch J, Daniel H and Wenzel U (2009) Feeding a ROS-generator to Caenorhabditis elegans leads to increased expression of small heat shock protein HSP-16.2 and hormesis. Genes Nutr 4, 59-67

8. Baik JH (2013) Dopamine signaling in food addiction: role of dopamine D2 receptors. BMB Rep 46, 519-526

9. Min S, Chung J (2016) Identification of a neural pathway governing satiety in Drosophila. BMB Rep 49, 137-138

10. Caldwell KA, Shu Y, Roberts NB, Caldwell GA and O'Donnell JM (2013) Invertebrate models of dystonia. Curr. Neuropharmacol 11, 16-29

11. Nall AH, Shakhmantsir I, Cichewicz K, Birman S, Hirsh J and Sehgal A (2016) Caffeine promotes wakefulness via dopamine signaling in Drosophila. Sci Rep 12, 20938

12. Brenner S (1974) The genetics of Caenorhabditis elegans. Genetics 77, 71-94

13. Maeda I, Kohara Y, Yamamoto M and Sugimoto A (2001) Large-scale analysis of gene function in Caenorhabidits elegans by high-throughput RNAi. Curr Biol 11, 171-176

14. Sharfer WR and Kentyon CJ (1995) A calcium-channel homologue required for adaptation to dopamine and serotonin in Caenorhabdits elegans. Nature 375, 73-78

15. Benedetto A, Au C, Avilla DS, Milatovic D and Aschner M (2010) Extracellular dopamine potentiates Mn-induced oxidative stress, lifespan reduction, and dopaminergic neurodegeneration in a BLI-3-dependent manner in Caenorhabditis elegans. PLos Genet 6, e1001084 